\title{
TOWARDS A NEW LHC INTERACTON REGION DESIGN FOR A LUMINOSITY UPGRADE
}

\author{
J. Strait ${ }^{+}$, M. Lamm, P. Limon, N.V. Mokhov, T. Sen, A.V. Zlobin, FNAL*, Batavia, IL, USA \\ O. Brüning, R. Ostojic, L. Rossi, F. Ruggiero, T. Taylor, H. ten Kate, CERN, Geneva, Switzerland \\ A. Devred, CEA/Saclay, Gif-sur-Yvette, France and CERN, Geneva, Switzerland \\ R. Gupta, M. Harrison, S. Peggs, F. Pilat, BNL*, Upton, NY, USA \\ S. Caspi, S. Gourlay, G. Sabbi, LBNL*, Berkeley, CA, USA
}

\section{Abstract}

After the LHC operates for several years at nominal parameters, it will be necessary to upgrade it for higher luminosity. Replacing the low- $\beta$ insertions with a higher performance design based on advanced superconducting magnets is one of the most straightforward steps in this direction. Preliminary studies show that, with magnet technology that is expected to be developed by early in the next decade, a factor of 2 to 5 reduction in $\beta^{*}$ could be achieved with new insertions, as part of an upgrade aimed at a factor of 10 luminosity increase. In this paper we survey several possible second generation LHC interaction regions designs, which address the expected limitations on LHC performance imposed by the baseline insertions.

\section{INTRODUCTION}

Although initial operation of the LHC is several years away, studies have begun for upgrades to extend its performance [1,2]. A luminosity upgrade towards $10^{35} \mathrm{~cm}^{-2}$ $\mathrm{s}^{-1}$ will be desired by the middle of the next decade after the LHC has operated for several years at its nominal parameters. The baseline LHC already pushes the limits of the state-of-the-art, and an extended R\&D program will be needed to ensure that the new technologies required for the upgrade, especially advanced superconducting magnets, are ready. In this paper we discuss several possible new interaction region (IR) designs which address potential limitations in the baseline LHC, and indicate the R\&D that must be done to aim towards higher luminosity.

\section{IR LAYOUTS}

Three major factors drive the designs of new IRs: minimizing $\beta^{*}$, minimizing the effects of long-range parasitic beam-beam interactions, and the large radiation power due to the $p p$ collisions $\left(9 \mathrm{~kW} /\right.$ beam at $\left.10^{35} \mathrm{~cm}^{-2} \mathrm{~s}^{-1}\right)$ directed towards the IRs. The first two point towards maximizing the magnet apertures and minimizing their distances to the IP, and the solutions to the third must be considered in every configuration.

Figures 1-5 show five sample IR layouts that address these issues in different ways and to different extents. The main parameters of these IRs are summarized in Table 1, which shows the distance from the IP to the first quadrupole, the quadrupole coil aperture $\left(D_{\text {quad }}\right)$, the mini-

\footnotetext{
*Work supported by the United States Department of Energy. +strait@fnal.gov
}

mum $\beta^{*}$ achievable consistent with physical aperture requirements, $\beta_{\max }$ for $\beta^{*}{ }_{\text {min }}$, and the strength $\left(\mathrm{B}_{\mathrm{D} 1}\right)$, length $\left(\mathrm{L}_{\mathrm{D} 1}\right)$, and coil aperture $\left(\mathrm{D}_{\mathrm{D} 1}\right)$ of the first dipole.

Preliminary studies [3] suggest that with increases in $\mathrm{J}_{\mathrm{c}}$ of $\mathrm{Nb}_{3} \mathrm{Sn}$ that are expected within the next 3-5 years, it may be possible to build quadrupoles with apertures up to $110 \mathrm{~mm}$ that operate, with $20 \%$ margin, at the same 200 $\mathrm{T} / \mathrm{m}$ as in the baseline IRs. This gradient with this aperture requires a 4-layer coil with outer radius sufficiently large to preclude its use in a dual-bore geometry with 194 $\mathrm{mm}$ spacing. In this study, we take $100 \mathrm{~mm}$ as an upper bound on the quadrupole aperture in a dual-bore magnet.

The minimum $\beta^{*}$ is set by the maximum possible beam size in the IR quadrupoles at $\beta_{\max }$. The required physical aperture in the baseline quadrupole triplet is estimated in Eqn. (5) in [1] as $1.1 \times(7.5+2 \times 9) \sigma_{\max }+2 \times 8.6 \mathrm{~mm}$, where $\sigma_{\max }$ is the maximum rms beam size, $7.5 \sigma$ is the minimum beam separation, $9 \sigma$ is the required envelope about each beam, the factor 1.1 accounts for $20 \% \beta$ beating, and $8.6 \mathrm{~mm}$ is the sum of mechanical tolerance and orbit errors. For beams passing on axis, the factor of $7.5 \sigma$ is dropped, and for off-axis orbits in the separation dipoles, the $7.5 \sigma$ term is replaced by the actual orbit offset. In determining $\beta_{\max }$ and $\beta^{*}{ }_{\min }$, the physical aperture is assumed to be $15 \mathrm{~mm}$ smaller than the coil diameter.

The field strength of the first dipole is given for the case of zero horizontal crossing angle. Generally the field is lower for non-zero crossing angles, as shown in the figures. The dipole coil diameter is computed according to the formulation above.

The simplest IR upgrade (Fig. 1) replaces the existing inner triplet with quadrupoles of the same strength and length, but larger aperture. A factor of 3 reduction in $\beta^{*}$ is possible, if all other parameters are held constant. However, other than using a shorter D1, this change doesn't reduce the number of parasitic collisions. If the beam

Table 1: IR Parameters

\begin{tabular}{|l|c|c|c|c|c|c|}
\hline & $\begin{array}{c}\text { Base- } \\
\text { line }\end{array}$ & Fig. 1 & Fig. 2 & Fig. 3 & Fig. 4 & Fig. 5 \\
\hline IP to Q1 (m) & 23 & 23 & 52.8 & 42.5 & 34 & 23 \\
\hline $\mathrm{D}_{\text {quad }}(\mathrm{mm})$ & 70 & 110 & 100 & 100 & 100 & 100 \\
\hline$\beta_{\min }^{*}(\mathrm{~cm})$ & 50 & 16 & 26 & 19 & 15 & 10 \\
\hline$\beta_{\max }(\mathrm{km})$ & 5 & 15 & 23 & 23 & 23 & 23 \\
\hline $\mathrm{B}_{\mathrm{D} 1}(\mathrm{~T})$ & 2.75 & 15.3 & 15 & 14.6 & 14.5 & 14.3 \\
\hline $\mathrm{L}_{\mathrm{D} 1}(\mathrm{~m})$ & 9.45 & 1.5 & 10 & 12 & 6 & 9 \\
\hline $\mathrm{D}_{\mathrm{D} 1}(\mathrm{~mm})$ & 80 & 110 & 135 & 165 & 75 & 105 \\
\hline
\end{tabular}




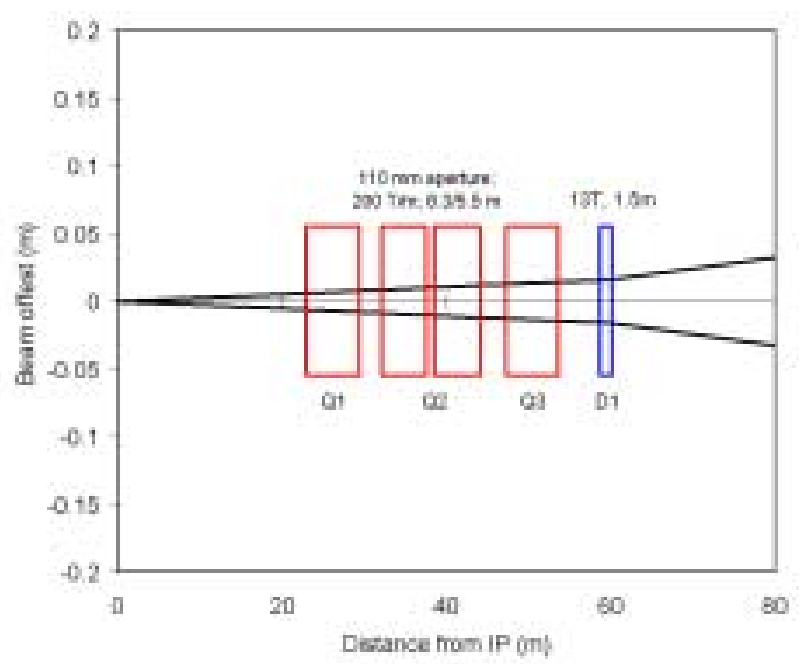

Figure 1: Quadrupole-first IR.

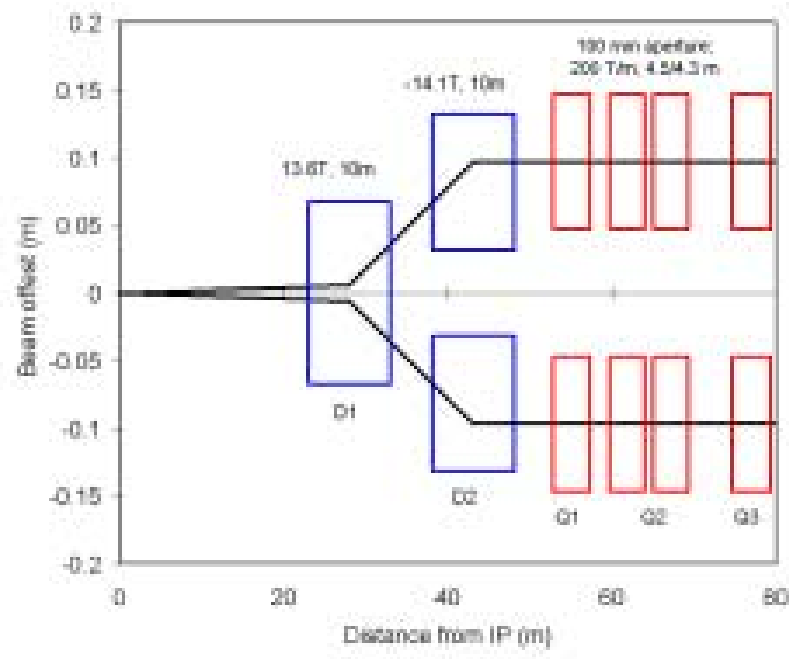

Figure 2: Dipoles-first IR.

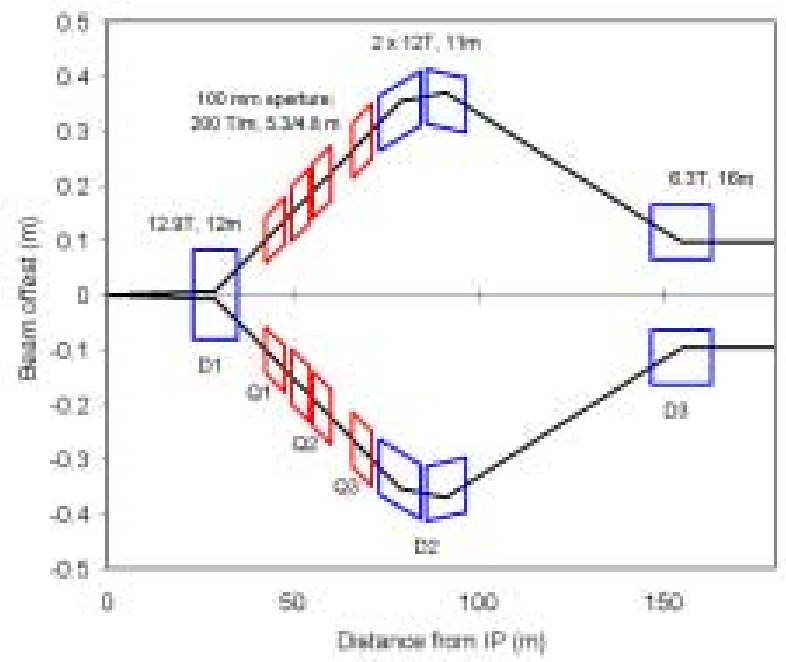

Figure 3: IR with quads between the separation dipoles.

current is increased to increase luminosity, then it is likely that the crossing angle will have to be increased, forcing an increase in $\beta^{*}$ to stay within the physical aperture.

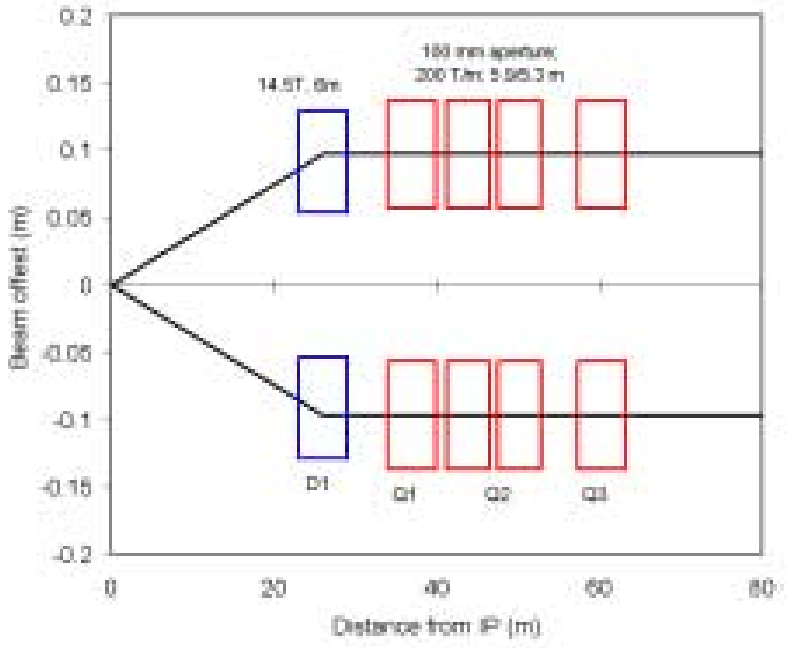

Figure 4: Dipole-first IR with large crossing angle.

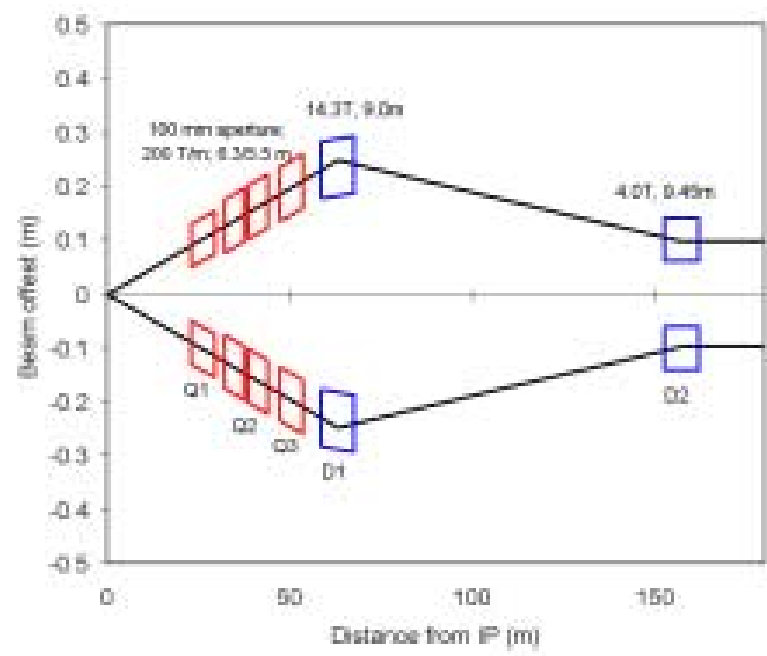

Figure 5: Quadrupole-first IR with large crossing angle.

Placing the separation dipoles between the IP and the quadrupoles (Fig. 2) reduces the number of parasitic collisions by more than a factor of three. Also, correction of quadrupole field errors is more robust, since the beams pass through the quadrupoles on axis and independent correction elements can be used for each beam. However, the quadrupoles are considerably farther from the IP, increasing $\beta_{\max }$ for a given $\beta^{*}$. The D1 also suffers very large energy deposition from collision debris, since the majority of the forward charged particles will be swept into it by the large magnetic field [4]. An added challenge is to maintain good field quality in the D2 with strong coupling between the two close, high-field apertures.

To maintain the benefits of the dipole-first layout, but reduce $\beta_{\max }$, the quadrupoles could be placed between the separation dipoles (Fig. 3), requiring dual-bore quadrupoles with non-parallel axes. The D2 and D3 restore the beams to a $194 \mathrm{~mm}$ spacing at the same distance as in the baseline. This layout allows a smaller $\beta^{*}$ than in Fig. 2, but the D1 is in the same radiation environment, and the feasibility of the non-parallel axis magnets and of dispersion suppression in this geometry have not been studied. 
The IRs in Figs. 1-3 assume that the crossing angle $\theta_{\mathrm{c}}$ is as small as possible, consistent with maintaining $>9 \sigma$ beam separation at all parasitic collisions. This is the solution if beam intensity is limited by factors other than the beam-beam effect. If the intensity can be increased to the beam-beam limit, then the luminosity is increased by increasing $\theta_{\mathrm{c}}$ and lengthening the bunches (including the "superbunch" option) [1,5]. This allows us to consider two large crossing angle layouts, shown in Figs. 4 and 5.

The first has a twin-aperture dipole first, then conventional dual-bore quadrupoles. The beam separation and the distance to the IP set $\theta_{\mathrm{c}}= \pm 3.7 \mathrm{mrad}$. The $\beta_{\text {min }}$ is as small as in Fig. 1. But this layout is practical only if the beam current can be increased sufficiently (or crab cavities are used to rotate the bunches). The aperture through which the outgoing beam passes is subject to similar radiation heating as the D1 in Figs. 2 and 3. In fact, the situation is more challenging since the forward going neutral particles may impinge on the magnet, if the aperture is not sufficient to allow them to pass through the magnet.

The layout in Fig. 5 aims non-parallel axis twinaperture quadrupoles directly at the IP. With the same optics as in Fig. 1 but in a dual-bore geometry, $\beta^{*}$ as small as $10 \mathrm{~cm}$ may be possible. As with Fig. 4, this is practical only if the beam current can be increased enough. As with Fig. 3, the non-parallel axis configuration must be studied for feasibility. And the first dipole is subject to similar radiation heating as the D1 in Fig 4.

\section{DISCUSSION}

The IR layouts presented here for a possible LHC luminosity upgrade are very preliminary, and much work remains to show that the estimated reductions in $\beta^{*}$, which are based on simple scaling rules for aperture and beambeam separation requirements, can be achieved when all constraints and realistic conditions are included.

Other accelerator systems must be upgraded if the reduction in $\beta^{*}$ is to translate into a corresponding increase in luminosity. If bunch intensity is limited below the beam-beam limit, then the bunches must be shortened to limit the luminosity loss due the crossing angle form factor. The required RF voltage grows as $\beta^{*}$ decreases, and it may or may not be feasible to take full advantage of $\beta^{*}<$ $25 \mathrm{~cm}$. Alternatively, crab cavities could rotate the bunches so they collide head-on in a transversely moving coordinate system. However, any imperfections in bunch manipulation will result in a transverse emittance blowup. To allow the bunch intensity to be increased to the beam-beam limit at larger crossing angles, many factors, some of which will be known only with operating experience, must be addressed. For example, the collimation or beam dump systems may need to be upgraded, or unexpected beam instabilities may need to be dealt with. A substantial new RF system would be required if the superbunch option were to be implemented.

The choice of IR layout for the luminosity upgrade will depend on developing an understanding of the LHC beams and which factors limit the luminosity. For exam- ple, the extent to which the parasitic collisions limit the performance will only be fully understood after experience with LHC beams. Successful development of proposed systems for compensating the beam-beam effect could affect the choice of IR layout. Finally, the upgrade scheme may be affected by the capabilities of the experiments, for example with regard to bunch structure.

Many questions specific to the IR magnet system must be addressed by vigorous R\&D. A few examples are:

- What is the maximum $\mathrm{D}_{\text {quad }}$ for $\mathrm{G}>200 \mathrm{~T} / \mathrm{m}$ ?

- What is the maximum $\mathrm{D}_{\text {quad }}$ in a dual-bore quadrupole with 194 mm spacing?

- Can dipoles be made to operate as high as $15 \mathrm{~T}$ in the extreme radiation environment at very high luminosity?

- How can the many $\mathrm{kW}$ of beam power be removed from the cryogenic magnets for a tolerable cost?

- Are non-parallel axis dual-bore quadrupoles feasible?

- What dispersion suppression scheme works in this case?

- Can good field quality be maintained over the full operating range in very high field, dual-bore dipoles with parallel field directions?

- Can triplet errors be adequately corrected given the very large $\beta$-functions?

Some of these questions can be answered by design studies, but most will require extended hardware R\&D.

\section{CONCLUSIONS}

A new IR design will be a key element in an LHC upgrade aimed at raising the luminosity by up to an order of magnitude. Several possible IR layouts exist which offer the possibility of reducing $\beta^{*}$ by up to a factor of five below the baseline value, and of reducing the number of long-range beam-beam collisions. Extensive R\&D must be done on superconducting magnets and other accelerator systems to determine if these ideas are feasible and to develop the technology to allow their implementation on the time scale required by the LHC physics program. Because the systems for the upgraded LHC must go well beyond the current state of the art, this $R \& D$ must start. Ultimately the performance of the baseline LHC, together with the results of this $R \& D$, will determine which of these IR designs will best serve to raise the luminosity.

\section{REFERENCES}

[1] O. Brüning, et al., LHC Luminosity and Energy Upgrade: A Feasibility Study, LHC Project Report 626, December 2002.

[2] T. Taylor, Superconducting Magnets for a Super LHC, EPAC 2002, Paris, France, p.129.

[3] A.V. Zlobin, et al., Aperture Limitations for $2^{\text {nd }}$ Generation $\mathrm{Nb}_{3} \mathrm{Sn}$ LHC IR Quadrupoles, PAC 2003, Portland, OR.

[4] N.V. Mokhov, et al., Energy Deposition Limits in a $\mathrm{Nb}_{3} \mathrm{Sn}$ Separation Dipole in Front of the LHC HighLuminosity Inner Triplet, PAC 2003, Portland, OR.

[5] F. Zimmermann, et al., Beam-Beam Interaction, Electron Cloud, and Intrabeam Scattering for Proton Super-Bunches, presented at PAC2003, Portland, OR. 